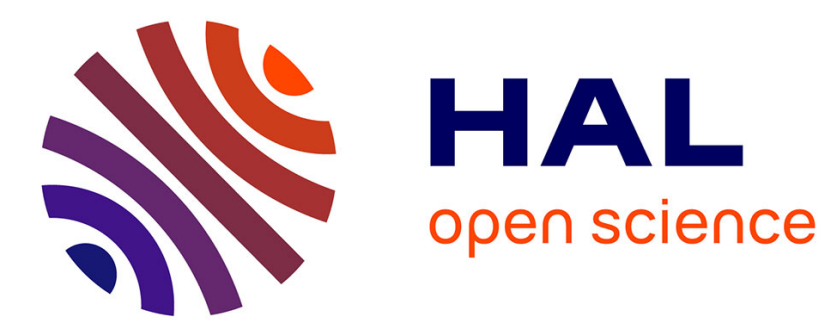

\title{
FORC signatures and switching-field distributions of dipolar coupled nanowire-based hysterons
}

\author{
A Pierrot, F Béron, T Blon
}

\section{To cite this version:}

A Pierrot, F Béron, T Blon. FORC signatures and switching-field distributions of dipolar coupled nanowire-based hysterons. Journal of Applied Physics, 2020, 10.1063/5.0020407 . hal-03382647

\section{HAL Id: hal-03382647 \\ https://hal.science/hal-03382647}

Submitted on 18 Oct 2021

HAL is a multi-disciplinary open access archive for the deposit and dissemination of scientific research documents, whether they are published or not. The documents may come from teaching and research institutions in France or abroad, or from public or private research centers.
L'archive ouverte pluridisciplinaire HAL, est destinée au dépôt et à la diffusion de documents scientifiques de niveau recherche, publiés ou non, émanant des établissements d'enseignement et de recherche français ou étrangers, des laboratoires publics ou privés. 


\title{
FORC signatures and switching-field distributions of dipolar coupled nanowire-based hysterons
}

\author{
A. Pierrot ${ }^{1}$, F. Béron ${ }^{2}$, T. Blon ${ }^{1, *}$ \\ ${ }^{1}$ Université de Toulouse, Laboratoire de Physique et Chimie des Nano-Objets, UMR 5215 INSA, CNRS, \\ UPS, 135 Avenue de Rangueil, F-31077 Toulouse,cedex 4, France \\ ${ }^{2}$ Universidade Estadual de Campinas, UNICAMP, Instituto de Física Gleb Wataghin, Rua Sergio \\ Buarque de Holanda 777, BR-13083859 Campinas, SP, Brazil \\ *corresponding author : thomas.blon@insa-toulouse.fr
}

\begin{abstract}
Analysis of first-order reversal curves (FORCs) is a powerful tool to probe irreversible switching events in nanomagnet assemblies. As in essence switching events are related to the intrinsic properties of the constituents and their interactions, resulting FORC diagrams contain much information that can be crosslinked and complex to deconvoluate. In order to quantify the relevant parameters that drive the FORC diagrams of arrays of perpendicularly magnetized nanomagnets, we present step-by-step simulations of assemblies of hysterons to determine the specific signatures related to different known inputs. While we explored the consequences of dipolar interactions using either mean field or magnetostatic approaches, we completed by taking the hysteron switching-field distribution (SFD) as either normal or log-normal. We demonstrated that the transition between FORC diagrams composed of an isolated interaction field distribution (IFD) and a wishbone shape operates via the SFD deviation, $\sigma_{H_{S W}}$, in the presence of a weakly dispersed interaction field. In the presence of a magnetostatic interaction field, the IFD profile is peaked and a coercive field distribution (CFD) sums to the IFD as $\sigma_{H_{S w}}$ increases. A transition between IFD+CFD and wishbone shapes is clearly demonstrated as a function of the interaction field deviation $\sigma_{H_{\text {int }}}$. In addition, we demonstrate that whatever the considered cases, $\sigma_{H_{s w}}$ can be quantitatively extracted from the FORC diagrams within an error inferior to $10 \%$. These findings are of interest for dipolar coupled perpendicularly magnetized nanomagnets, as in assemblies of magnetic nanowires and nanopillars, as well as bit patterned media.
\end{abstract}




\section{Introduction}

The extraction of the individual behaviour of entities based on the measurements of their collection is a fundamental problem encountered in many fields such as biology, sociology, ethology, physics, etc. Concerning hysteretic processes, Mayergoyz introduced an approach for the determination of the weight function of a set of hysteresis operators [1], typically based on the Preisach model of hysterons describing magnetic hysteresis [2]. Experimentally, this approach resides in the measurement of first-order reversal curves (FORCs) [3,4], which consist in saturating a magnetic sample in the positive direction, then decreasing the magnetic field $H$ up to a field $H_{r}$, in order to measure the magnetization $M$ of the corresponding FORC between $H_{r}$ and the positive saturation. Typically, between one and two hundreds of $H_{r}$ values are chosen to fill the major hysteresis loop (MHL) to compute the FORC diagram using the second order mixed derivative :

$$
\rho_{F O R C}\left(H, H_{r}\right)=-\frac{1}{2} \frac{\partial^{2} M\left(H, H_{r}\right)}{\partial H_{r} \partial H},\left(H \geq H_{r}\right) \quad \text { (Eq. 1) }
$$

FORC analysis is now widely used in several laboratories because of its implementation simplicity [3,5]. Focusing on magnetic systems, FORC technique has been used to investigate diverse systems such as rock and mineral magnetism $[3,6,7,8]$, magnetic media $[9,10,11,12]$, GMR spin valves [13], magnetic tunnel junctions [14], thin films $[15,16,17,18]$, magnetic multilayers with perpendicular magnetic anisotropy [19,20,21,22,23,24], magnetic dot/pillar assemblies [25,26,27], permanent magnets [28,29], exchange spring magnets [30], and nanowire assemblies $[31,32,33,34,35,36,37,38,39,40,41,42]$, since isolated small diameter nanowires behave as basic hysterons. However, the understanding of the resulting FORC diagrams is not straightforward as it does not strictly represent the Preisach distribution. Therefore, simulations are mandatory to qualitatively and quantitatively read the relevant information $[5,35,43,44,45,46,47,48,49]$.

One of the key features of a nanomagnet assembly is the switching field distribution (SFD). It is the figure of merit of recording media as it drives magnetic stability and achievable recording density. In a dense assembly where dipolar interactions are important, the SFD extraction represents a complex problem. Different methods have been proposed $[50,51]$, each one introducing inherent assumptions. The FORC approach, with its ability to map switching events, interactions, multiphase systems, microscopic behaviour, etc, would be a powerful tool in quantifying the SFD [52,53]. The drawback of such a multitude of attainable properties is that crosslinked behaviours can alter its quantification.

Here, we present systematic simulations of assemblies of nanowire-based hysterons in order to determine the specific FORC signatures related to: (i) dipolar interactions, using both mean field and magnetostatic approaches, with tuned amplitude and deviation; (ii) hysteron SFD, for both normal and log-normal distributions, also varying amplitude and deviation. First, we consider the case of identical hysterons (part A) interacting via (i) a mean demagnetizing dipolar field, and (ii) a calculated magnetostatic interaction field. Normal and log-normal switching field distributions (SFD) are then introduced in both cases (part B). The effect of the SFD standard deviation is investigated in both in the presence of a mean or magnetostatic interaction field, as well as those arising from the interaction field amplitude and deviation. Finally, we demonstrate that the SFD deviation can be extracted from the FORC diagrams with an error inferior to $10 \%$, and this, whatever the SFD shape and interaction field regime.

\section{Model}

Assemblies of $N$ hysterons were considered with determined switching-field value $\left\langle H_{s w}\right\rangle$ and standard deviation $\sigma_{H_{S W}}$. All thermal effects and exchange interactions between hysterons were neglected. The modelled systems correspond to 2D nanowire arrays with an applied magnetic field 
perpendicular to the assembly plane (i.e., along the nanowire axis, $z$ ). As a consequence, the nanowires are only magnetized either up or down, yielding an interaction field remaining along the nanowire axis as well.

For a mean interaction field, it is always opposite to the averaged magnetization $M_{z}$. Therefore, we considered a mean interaction field of demagnetizing type (moving Preisach model [54]):

$$
\mu_{0} H_{\text {int }, z z}=\alpha_{m} \frac{M_{z}}{M_{S}}
$$

where $\mu_{0}$ is the Bohr magneton, $M_{S}$ the saturation magnetization, and with $\alpha_{m}<0$ ( $\alpha_{m}$ expressed here in T). Since the resulting mean interaction field is uniform, all $N=1000$ hysterons are submitted to the same value, i.e. there is no dispersion $\left(\sigma_{\text {Hint }}=0\right)$.

On the other side, for a magnetostatic dipolar field, the hysterons were taken as spatially localized and referring to nanowires of diameter $D$ and length $L$. Calculations were performed considering a planar triangular assembly with lattice vectors $\vec{a}=\left(\begin{array}{c}d+D \\ 0\end{array}\right)$ and $\vec{b}=\left(\begin{array}{c}(d+D) / 2 \\ (d+D) \sqrt{3} / 2\end{array}\right)$, with $d$ the edge to edge inter-hysteron distance. Unless stated, the dimensions used in the simulations are $D=7 \mathrm{~nm}, L=60 \mathrm{~nm}$, and $d=6 \mathrm{~nm}$, with $N=1681$ hysterons. The interaction field was calculated using the magnetostatic field between neighbouring nanowires [55]:

$$
\mu_{0} H_{\text {int }, z z}=\mu_{0} D^{2} L M_{S} \sum_{i \neq j}\left(\frac{1}{\left(\rho_{i j}^{2}+L^{2}\right)^{3 / 2}}\right)
$$

where $\rho_{i j}$ is the distance between two $i$ and $j$ nanowire-based hysterons. The sum was calculated over all hysterons and updated after every switching event. Such a calculated interaction field allows considering the case of an inhomogeneous interaction field. In particular, it scales with the number of considered hysterons. Since the interaction field is higher for hysterons located at the assembly centre than at its periphery, modulating the hysteron number, i.e. the size of the assembly, introduces a deviation $\sigma_{\text {Hint }}$ of the interaction field.

Whatever the considered interaction field, it sums to the applied external magnetic field $H_{\text {ext }}$, yielding a total field acting on each hysteron $H_{\text {tot }}=H_{\text {ext }}+H_{\text {int }}$ (Eq. 4). As the applied field changes quasistatically, the hysterons are flipped one by one if $H_{t o t}>H_{s w}$, then $H_{\text {int, } z z}$ is updated and all new possible switchings are examined, until the equilibrium magnetization is reached [43].

For each specific case described below, 80 FORCs were calculated. The FORC diagrams were computed (Eq. 1) and plotted using the interaction field axis $H_{\text {int }}=-\frac{H+H_{r}}{2}$ and the hysteron halfwidth axis $H_{C}=\frac{H-H_{r}}{2}$ (Supplementary Material, part B) and normalized with respect to the maximum value $\rho_{F O R C}^{\max }$ of the corresponding diagram. Negative $\rho_{F O R C}$ values were not considered here.

\section{Results}

\section{A. Identical hysterons}

Starting from the simplest but unrealistic case, we first consider identical hysterons, i.e. hysterons with identical switching field $H_{S w}$. In this case, the intrinsic switching field of the $N$ hysterons is set to $\mu_{0} H_{s w}=300 \mathrm{mT}$, with no switching field distribution $\left(\sigma_{H_{S W}}=0\right)$. The effect of a mean interaction field is first presented, followed by a magnetostatic field, both as previously described. 


\section{Mean interaction field}

The resulting major hysteresis loop (MHL, inset of Fig. 1a)) is clearly sheared due to demagnetizing interaction. Its differential susceptibility, $d M / d H$, remains constant during the magnetization reversal, with $\left(d\left(M / M_{s}\right) / d\left(\mu_{0} H\right)\right)^{-1}=\alpha_{m}$. The resulting FORC signature is a linear distribution of $\rho_{F O R C}$ strictly parallel to the $H_{\text {int }}$ axis (Fig. 1(a)) [56]. It originates from the $H_{\text {int }}$ variation of (Eq. 2) during the major hysteresis loop, with $-\alpha_{m} \leq \mu_{0} H_{\text {int }} \leq \alpha_{m}$. As a consequence, this FORC feature is usually referred as the interaction field distribution (IFD). The profile along the IFD is constant in height and extrema correspond to $\mu_{0} H_{\text {int }}^{\text {sat }}= \pm \alpha_{m}$ (Fig. 1(b)). Moreover, the crossing point between $\rho_{F O R C}$ and $H_{C}$ axis, is $H_{C}^{M H L}=H_{S W}$ in this case.
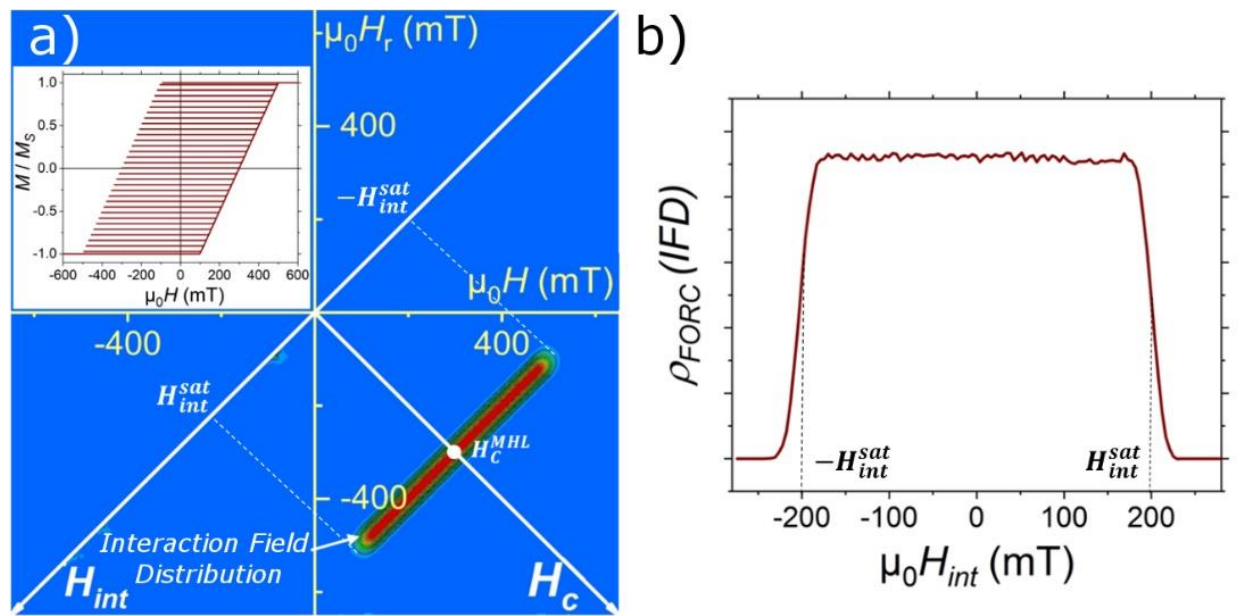

Figure 1: Identical hysterons ( $N=1000$ hysterons, $\mu_{0} H_{s w}=300 \mathrm{mT}, \sigma_{H_{S w}}=0$ ) submitted to a mean interaction field ( $\alpha_{m}=-200 \mathrm{mT}$ ). a) FORC diagram calculated from the simulated curves shown in inset. b) Profile along the $H_{\text {int }}$ axis of the interaction field distribution (IFD).

\section{Magnetostatic interaction field}

Contrary to the mean interaction field that is homogeneous, using a magnetostatic interaction field yields a MHL differential susceptibility that is not constant during the magnetization reversal (inset of Fig. 2(a)). The FORC diagram still exhibits an IFD, but with an amplitude that strongly varies (Fig. 2(b)). This characteristic can be considered as the FORC signature of hysterons interacting in an inhomogeneous interaction field, in opposition to the homogeneous interaction regime exhibiting IFD flat profile (Fig. 1(b)). Numerous FORC measurements performed on nanowire assemblies display such a signature $[31,33,42,56,57,58,59,60,61,62,63,64,65]$. Previous simulations of hysteron assemblies in the moving Preisach model indicated that IFD profiles flatten when increasing $\left|\alpha_{m}\right| / \sigma_{\text {int }}[66]$, in agreement with the data reported here. As for the mean interaction field, the crossing point between $\rho_{\text {FORC }}$ and $H_{C}$ axis, is $H_{C}^{M H L}=H_{S W}$. 

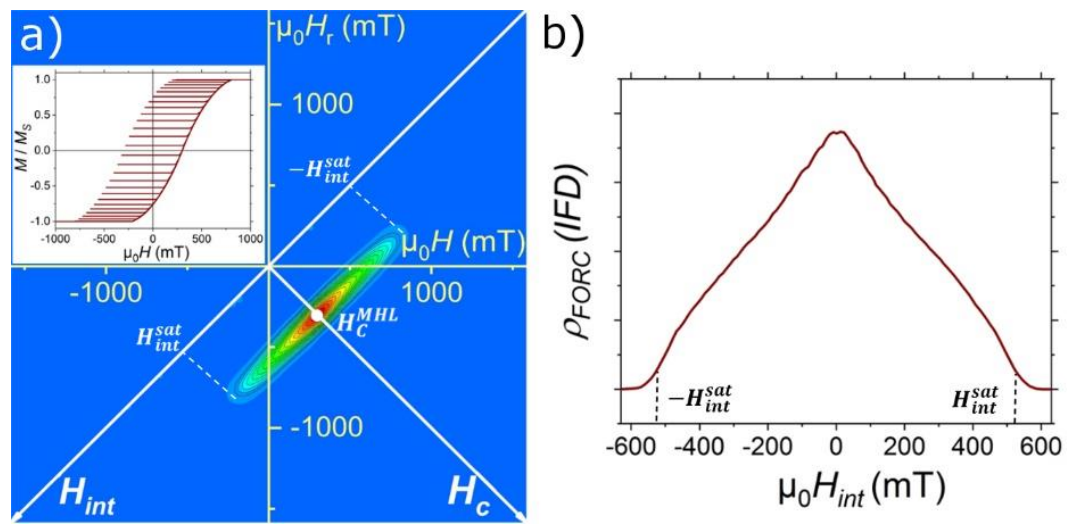

Figure 2 : Identical hysterons ( $N=961$ hysterons, $\left.\mu_{0} H_{s w}=300 \mathrm{mT}, \sigma_{H_{S w}}=0\right)$ submitted to a magnetostatic interaction field. a) FORC diagram calculated from the simulated curves shown in inset. b) IFD profile along the $H_{\text {int }}$ axis.

\section{B. Dispersed hysterons}

\section{Without interaction}

Before analysing the complex cases of hysteron assemblies presenting switching field distribution (SFD), it is important to understand the behaviour modifications caused without interaction. Introducing a SFD into an assembly of non-interacting hysterons organizes the switching events from the less coercitive to the most coercitive hysteron. For both normal and log-normal SFD, the resulting FORC signature is a $\rho_{F O R C}$ distribution aligned along the $H_{C}$ axis (Fig. 3). As expected, the associated $\rho_{F O R C}$ profiles along the $H_{C}$ axis are found identical to the normal and log-normal SFD introduced into the simulations. Fits of the $\rho_{F O R C}$ profile give $H_{c}^{\text {mean }}=297 \mathrm{mT}$ and $\sigma_{H_{S w}}^{F O R C}=51 \mathrm{mT}$ for the normal SFD (Fig. 3(b)). For the log-normal SFD (Fig. 3(d)), $H_{c}^{M H L}$ is equal to the fitted median value $H_{c}^{\text {median }}=299 \mathrm{mT}$, and $\sigma_{H_{S w}}^{F O R C}=110 \mathrm{mT}$. These values are consistent with the introduced ones $<\mu_{0} H_{s w}>=300 \mathrm{mT}$ and $\sigma_{H_{s w}}=50 \mathrm{mT}$ (normal) and $\sigma_{H_{s w}}=110 \mathrm{mT}$ (log-normal).
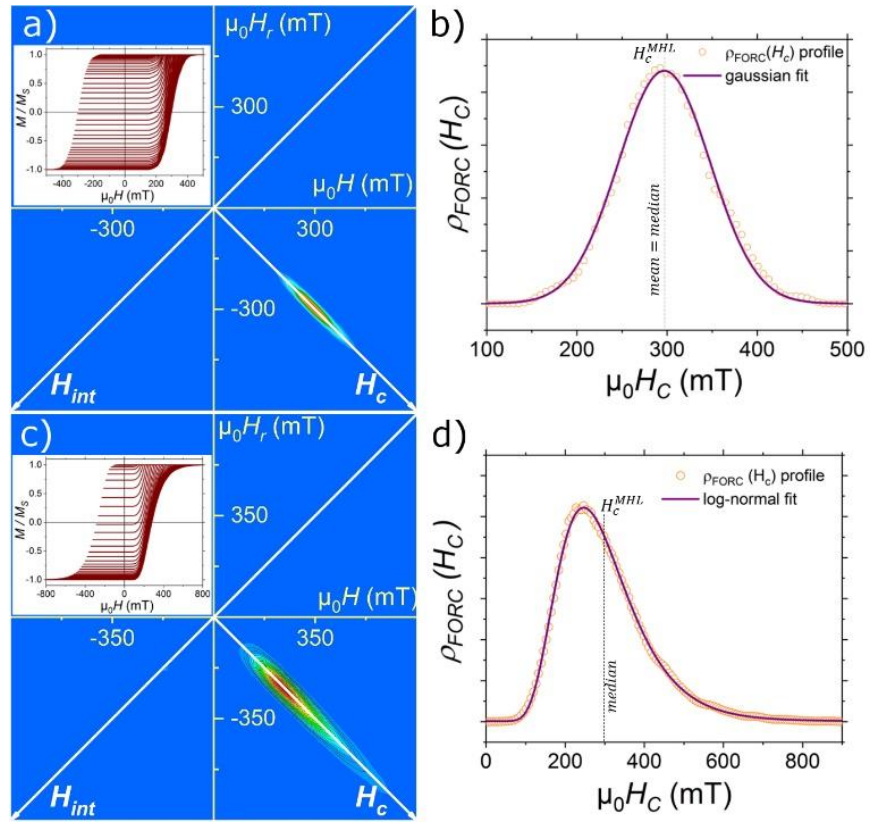

Figure 3: Dispersed non-interacting hysterons ( $N=1000$ hysterons, $\left.<\mu_{0} H_{s w}>=300 \mathrm{mT}\right)$ with (a)-(b) a normal SFD $\left(\sigma_{H_{S w}}=50 \mathrm{mT}\right)$ and (c)-(d) log-normal SFD $\left(\sigma_{H_{S w}}=110 \mathrm{mT}\right)$. (a)-(c) FORC diagrams calculated from the simulated curves shown in inset. (b)-(d) Profiles of $\rho_{F O R C}$ along the $H_{C}$ axis and corresponding fits. 


\section{Interactions and the switching field distribution}

When assemblies of moderately dispersed hysterons are interacting, the resulting FORC diagrams are similar to the identical hysteron cases. Indeed, for both mean (Fig. 4(a)-(b)) and magnetostatic interaction fields (Fig. 4(c)-(d)), the linear IFD persists with a width always equals to $2 H_{\text {int }}^{\text {sat }}$. Additionally, the $\rho_{F O R C}$ profiles along $H_{\text {int }}$ remain flat and peaked for mean and magnetostatic interaction fields, respectively. In all cases, increasing $\left\langle H_{s w}>\right.$ only shifts the IFD along the $H_{c}$ axis (See Supplementary Material, part A, Figs. S1-S2). However, despite these similarities, the hysteron SFD induces modification in the FORC diagrams. Since the interaction field nature alters differently the FORC results arising from dispersed hysterons, the impact of varying the hysterons non-uniformity is investigated separately.

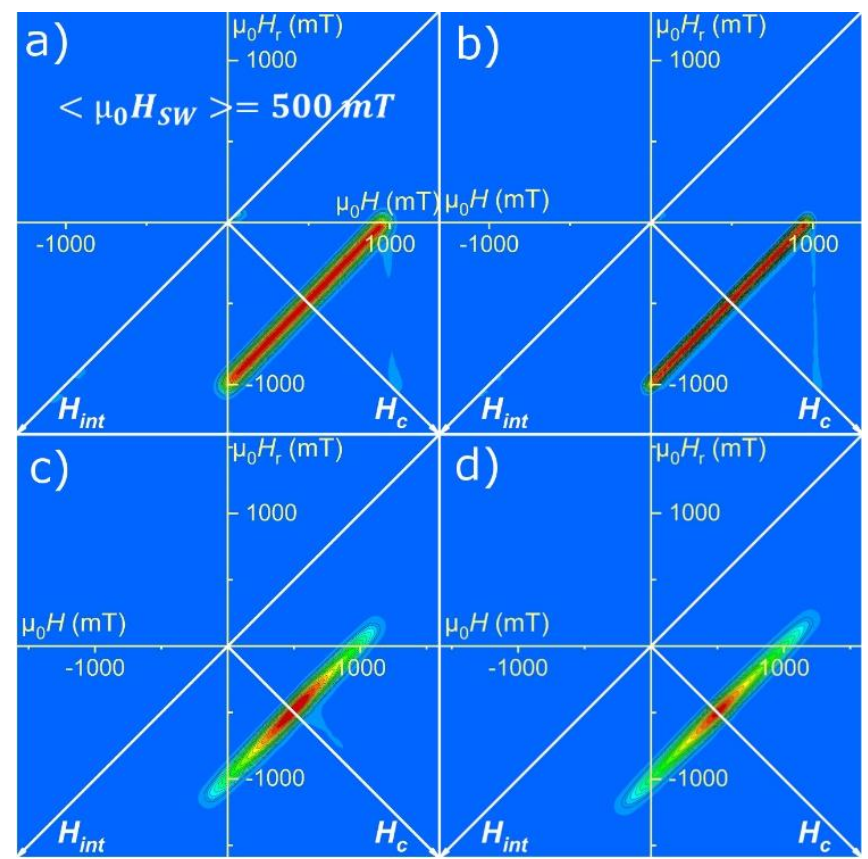

Figure 4 : FORC diagrams calculated for assemblies of hysterons interacting in a (a)-(b) mean interaction field or (c)-(d) magnetostatic interaction field for (a)-(c) normal and (b)-(d) log-normal switching field distributions. $<\mu_{0} H_{s w}>=500 \mathrm{mT}, \sigma_{H_{S w}}=10 \mathrm{mT}, \alpha_{m}=-500 \mathrm{mT}\left(\mu_{0} H_{\text {int }}^{\text {sat }}= \pm 500 \mathrm{mT}\right)$. FORC diagrams calculated with various $<\mu_{0} H_{s w}>$ values are presented in Figs. S1 and S2 of Supplementary Material.

\section{a. Mean interaction field}

When increasing $\sigma_{H_{S w}}$, i.e. nanowires less uniform, Figure 5 (normal SFD) and S3 (log-normal SFD, Supplementary Material) evidence the progressive transition occurring between an IFD FORC diagram at low $\sigma_{H_{s w}}$ and to a FORC diagram generally called "wishbone" [26] at large $\sigma_{H_{s w}}$. This is coherent with previous simulations where the wishbone shape was simulated in assemblies of distributed hysterons with mean field interactions [26]. Indeed, the wishbone shape is commonly encountered in assemblies of magnetic pillars or nanowires, as well as in thin films with perpendicular anisotropy [9][10][26][41][42][67]. 

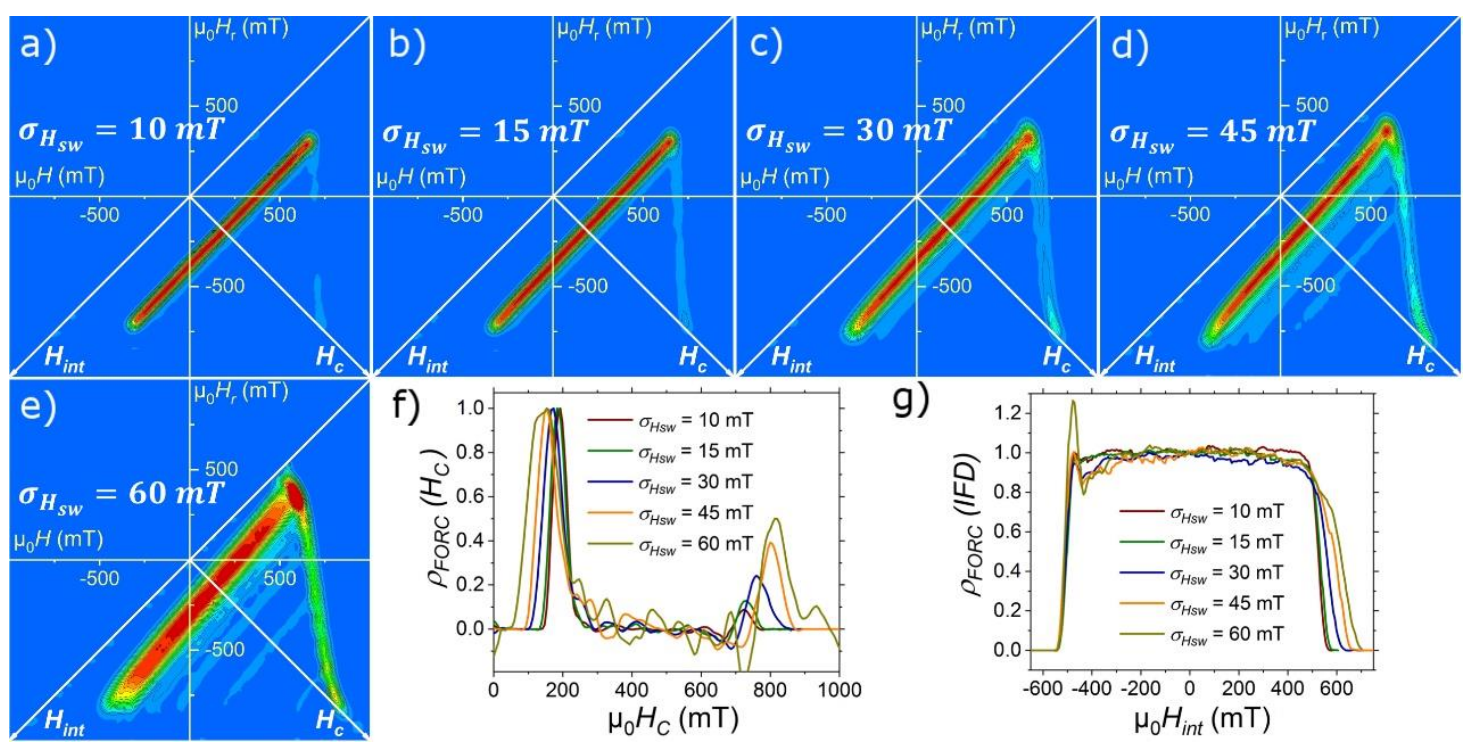

g)

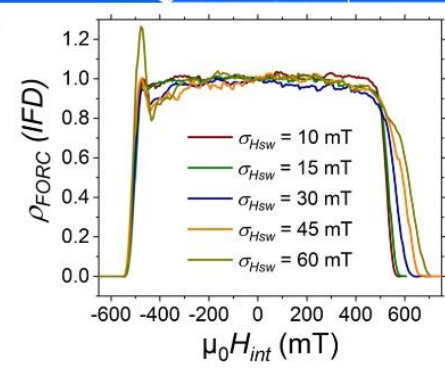

Figure 5: (a)-(e) FORC diagrams calculated for assemblies of hysterons presenting a normal SFD and interacting in a mean interaction field. $<\mu_{0} H_{s w}>=200 \mathrm{mT}, 10 \leq \sigma_{H_{S W}} \leq 60 \mathrm{mT}, \alpha_{m}=-500 \mathrm{mT}$ $\left(\mu_{0} H_{\text {int }}^{\text {sat }}= \pm 500 \mathrm{mT}\right.$ ). Profiles of (f) $\rho_{F O R C}$ along $H_{c}$ axis and (g) along $H_{\text {int }}$ axis for different $\sigma_{H_{S w}}$ (curves are normalized with respect to the $\rho_{F O R C}$ value at $H_{\text {int }}=0$ ). Corresponding FORC diagrams calculated for a lognormal SFD are presented in Figs. S3 of Supplementary Material.

This specific shape, shown in more details in Figure 6 , is the signature of a dispersed assembly interacting in a mean demagnetizing field. The $A B$ branch of Fig. 6 is the IFD previously described, for which the profiles remain flat (see Figs. $5(\mathrm{~g})$ and $\mathrm{S} 3(\mathrm{~g})$ ), as expected for a mean interaction field. However, it can be seen that it is no longer parallel to the $H_{\text {int }}$ axis as soon as $\sigma_{H_{s w}}$ increases, as frequently observed experimentally [9][26][41][42][67]. On the other side, the $A C$ branch, which could only be evidenced in the presence of a SFD, contains the last switching events that occur just before reaching saturation in a given FORC. As explained in details in part B of Supplementary Material, the most important aspect is that the switching events producing the $A C$ branch do not correspond to intrinsic switching values of hysterons, but to hysteron widths artificially enhanced by interactions.

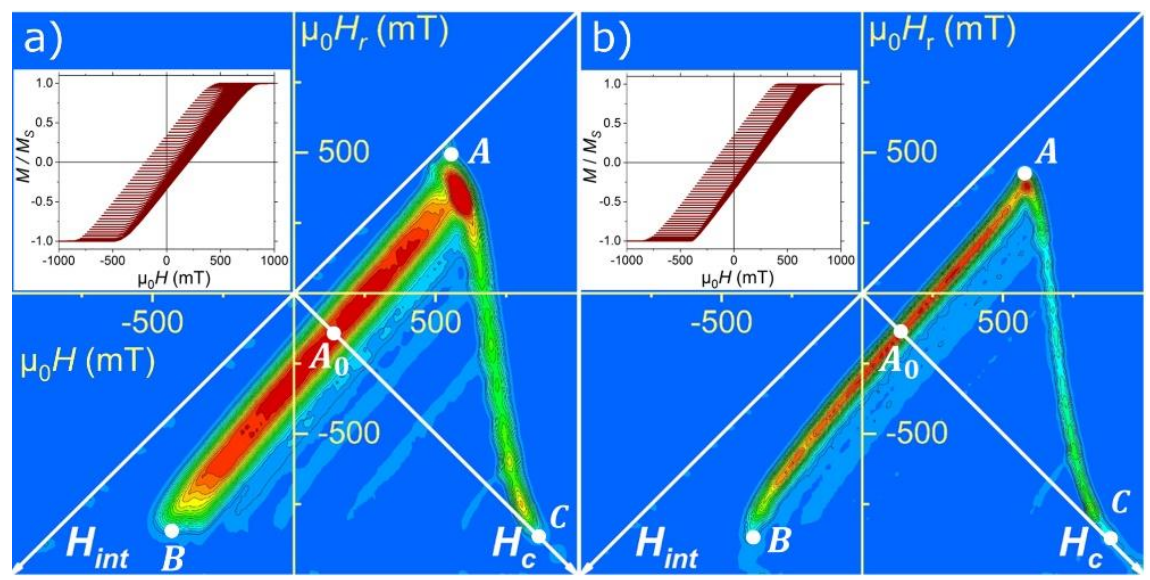

Figure 6 : Larger view of the wishbone shape obtained for a (a) normal (Fig. 5(e)) and (b) log-normal (Fig. S3(e)) SFD. $<\mu_{0} H_{s W}>=200 \mathrm{mT}, \sigma_{H_{s W}}=60 \mathrm{mT}, \alpha_{m}=-500 \mathrm{mT}$ ). Insets are the corresponding simulated FORCs.

Quantitative data such as the SFD deviation can be extracted from these FORC diagrams. Prosaically, based on the FORC diagram analysis of dispersed non-interacting hysterons (Fig. 3), profiles of $\rho_{F O R C}$ along $H_{c}$ axis (Fig. 5(f)) could be fitted to extract both the mean and deviation values, $H_{C}^{\rho_{F O R C}\left(H_{c}\right)}\left(A_{0}\right.$ 
point, Fig. 6) and $\sigma_{\text {fit }}^{\rho_{F O R C}\left(H_{C}\right)}$, respectively. Such values, reported in Table 1 (normal SFD, Fig. 5) and Table S1 (log-normal SFD, Fig. S3), clearly differ from the $\left\langle H_{S W}\right\rangle$ and $\sigma_{H_{S W}}$ values introduced in the simulations. Therefore, even though they follow the same tendency, it indicates that a $\rho_{F O R C}\left(H_{C}\right)$ profile does not represent the switching field distribution. In particular, one can note than $<H_{c}^{\rho_{F O R C}\left(H_{c}\right)}>$ decreases with $\sigma_{H_{s w}}$ due to the progressive tilt of the IFD.

\begin{tabular}{|c|c|c|c|c|c|}
\hline $\begin{array}{c}<H_{s w}> \\
(\mathrm{mT})\end{array}$ & $\begin{array}{c}\sigma_{H_{S W}} \\
(\mathrm{mT})\end{array}$ & $\begin{array}{c}H_{c}^{\rho_{F O R C}\left(H_{C}\right)} \\
(\mathrm{mT})\end{array}$ & $\begin{array}{c}\sigma_{f i t}^{\rho_{F O R C}\left(H_{c}\right)} \\
(\mathrm{mT})\end{array}$ & $\begin{array}{c}H_{c}^{\text {mean }} \\
(\mathrm{mT})\end{array}$ & $\begin{array}{c}\sigma_{H_{S W}}^{F O R C} \\
(\mathrm{mT})\end{array}$ \\
\hline 200 & 10 & 191 & $19.1(91 \%)$ & $204(2 \%)$ & $10.8(8 \%)$ \\
\hline 200 & 15 & 184 & $20.6(37 \%)$ & $196(2 \%)$ & $15.9(6 \%)$ \\
\hline 200 & 30 & 146 & $22.5(25 \%)$ & $202(1 \%)$ & $29.6(1.3 \%)$ \\
\hline 200 & 45 & 132 & $23.4(48 \%)$ & $205(2.5 \%)$ & $45.9(2 \%)$ \\
\hline 200 & 60 & 125 & $36.5(39 \%)$ & $205(2.5 \%)$ & $58.2(3 \%)$ \\
\hline
\end{tabular}

Table 1: Main properties extracted from FORC diagrams calculated for hysteron assemblies of presenting a normal SFD and interacting in a mean interaction field (Fig. 5).

$\left.<H_{S W}\right\rangle$ and $\sigma_{H_{S W}}$ are the mean switching field and deviation values of the SFD introduced in simulations, $H_{c}^{\rho_{F O R C}\left(H_{C}\right)}$ and $\sigma_{f i t}^{\rho_{F O R C}\left(H_{C}\right)}$ are the mean and deviation values deduced from $H_{c}$ axis profile fitting (Fig. $5\left(\right.$ f)). $H_{c}^{\text {mean }}$ is the $H_{c}$ value read on FORC diagrams at point $B . \sigma_{H_{S w}}^{F O R C}$ is the SFD deviation deduced from the FORC diagram and Eq. 5. The discrepancies with respect to the values introduced in simulations are indicated in parentheses.

However, the SFD parameters can be successfully estimated exploiting the $A, B$ and $C$ point coordinates in the $\left(H_{c}, H_{\text {int }}\right)$ plane $[5,68]: A:\left(H_{c}^{\text {min }} ;\left|H_{\text {int, } F O R C}^{\text {sat }}\right|\right), B:\left(H_{c}^{\text {mean }} ; H_{c}^{\text {mean }}+H_{c}^{\text {min }}-\right.$ $\left.\left|H_{\text {int }, F O R C}^{\text {sat }}\right|\right), C:\left(H_{c}^{\max }+\left|H_{\text {int,FORC }}^{\text {sat }}\right| ; 0\right)$. As outlined by A. Stancu et al. [5], estimation of the SFD deviation $\sigma_{H_{S w}}$ of a normal SFD can be obtained using $\sigma_{H_{S w}}^{F O R C}=\frac{<H_{c}^{\text {mean }}>-H_{c}^{\min }}{3}$ (Eq. 5), as $99.7 \%$ of variables in a normal distribution are contained in a $\pm 3 \sigma$ width from average value.

For a log-normal distribution, the $A, B, C$ point coordinates become : $A:\left(H_{c}^{\text {min }} ;\left|H_{\text {int }, F O R C}^{\text {sat }}\right|\right), B:\left(H_{c}^{\text {median }} ; H_{c}^{\text {median }}+H_{c}^{\text {min }}-\left|H_{\text {int }, F O R C}^{\text {sat }}\right|\right), C:\left(H_{c}^{\text {max }}+\left|H_{\text {int }, F O R C}^{\text {sat }}\right| ; 0\right)$. $\sigma_{H_{S w}}^{F O R C}$ can be estimated following the same approach of identifying the $\left(\left\langle H_{c}^{\text {mean }}\right\rangle-H_{c}^{\text {min }}\right)$ interval as a function of the log-normal variance to deduce $\sigma_{H_{S W}}^{F O R C}$ as equals to the variance square root (see Supplementary Material, part C). Tables 1 and $\mathrm{S} 1$ show that the $A, B, C$ points are good indicators to retrieve the hysteron intrinsic properties: the $H_{c}^{\text {mean }}$ (normal) and $H_{c}^{\text {median }}$ (log-normal) values extracted at point $B$ corresponds to $<H_{S w}>$ and $H_{C}^{M H L}$, while $\sigma_{H_{S w}}^{F O R C}$, estimated from $H_{c}^{m e a n}$ (normal) or $H_{c}^{\text {median }}$ (log-normal) and $H_{c}^{\min }$ (at point $A$ ), yields the adequate value of $\sigma_{H_{s w}}$ with errors less than $10 \%$ for both normal and log-normal SFDs. 


\section{b. Magnetostatic interaction field}

In the case of a magnetostatic interaction field, (Fig. 7), the switching field distribution induces an additional branch parallel to the $H_{C}$ axis where $\rho_{F O R C} \gtrsim 0$, sometimes referred as the coercive field distribution (CFD) [5]. Indeed, for both normal (Fig.7) and log-normal (Fig. S4) SFDs, FORC diagram are composed of an IFD and such a CFD. This last distribution is enhanced with $\sigma_{H_{S W}}: \rho_{F O R C}$ increases into the $A A_{0} C$ triangle, and particularly along the $A_{0} C$ segment. This can be understood following the wishbone shape description (part B of Supplementary Material): in the presence of a SFD, the hysteron width $\left(H_{c}^{i}\right)$ can be artificially increased due to interaction. In the presence of a mean interaction field, the $A C$ segment arises from hysterons whose switchings $u p \rightarrow$ down (just before the $i^{\text {th }}$ FORC) and down $\rightarrow$ up (during the $i^{\text {th }}$ FORC) occur at different $|M|$ values as a result of the total field scanning of the SFD. Therefore, it generates enhancement of both bias $\left(H_{u}^{i}\right)$ and width $\left(H_{c}^{i}\right)$ of the hysteron (Eq. S3 and S4 of Supplementary Material). On the opposite, in the presence of a magnetostatic interaction field, the $A C$ segment is almost missing, while the strong presence of the CFD on the $A_{0} C$ segment reveals mainly width $\left(H_{c}^{i}\right)$ enhancement in this case. It arises from the fact that both switchings of the $i^{\text {th }}$ hysteron (up $\rightarrow$ down and down $\rightarrow$ up) happen for nearly equal $|M|$ values, leading to $H_{u}^{i}=0$ (Eq. S1 of Supp. Mat.), which is a direct consequence of the local magnetostatic interaction field being nonuniform. Hence, switching events are guided not only by the hysteron SFD, but are also strongly affected by the local environment, i.e. the $H_{\text {int }}$ local value. Such a point is addressed in more details in Sect. $3 \mathrm{~b}$, introducing the deviation of the interaction field, $\sigma_{H_{i n t}}$. Nonetheless, this dependence on the interaction field local value explains why the IFD profiles (Fig. $7(\mathrm{~g})$ and $\mathrm{S} 4(\mathrm{~g})$ ) keep the peaked feature associated to the presence of a nonuniform interaction field, without clear distinction between the simulated hysteron distributions.
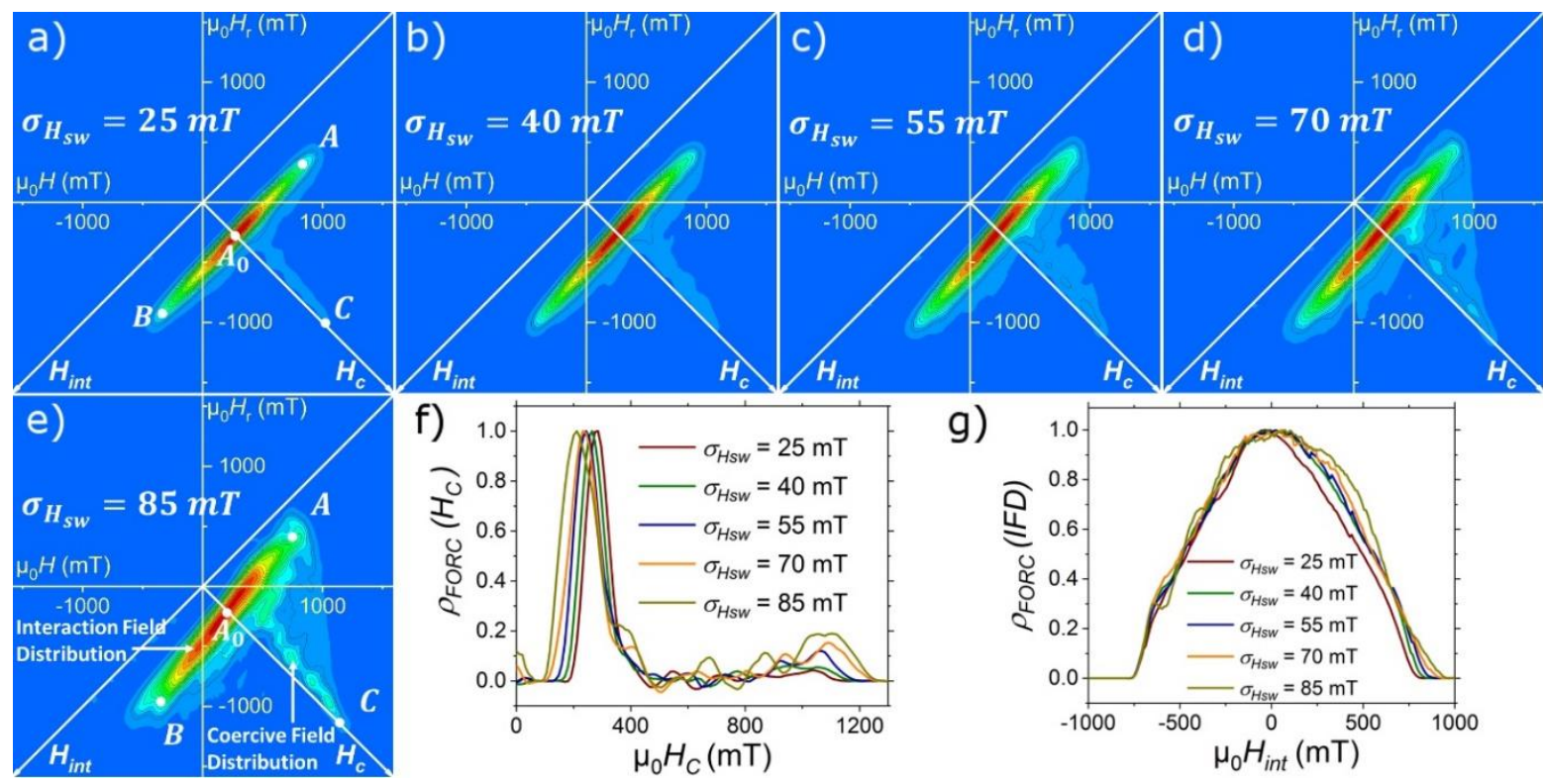

Figure 7: (a)-(e) FORC diagrams calculated for assemblies of hysterons presenting a normal SFD and interacting in a magnetostatic interaction field $<\mu_{0} H_{s w}>=300 \mathrm{mT}, 25 \leq \sigma_{H_{s w}} \leq 85 \mathrm{mT},<\mu_{0} H_{\text {int }}^{\text {sat }}>\cong$ $\pm 500 \mathrm{mT}$. (f) Profiles of $\rho_{F O R C}$ along $H_{C}$ axis for different $\sigma_{H_{S W}}$. (g) Profiles of $\rho_{F O R C}$ along the IFD.

Following the analysis previously detailed for a mean interaction field, one can observe in Tables 2 (normal SFD) and S2 (log-normal SFD) that the resulting $\rho_{F O R C}\left(H_{c}\right)$ profiles in the presence of a magnetostatic interaction field also do not directly represent the switching field distributions. Despite following a similar tendency, both $<H_{c}^{\rho_{F O R C}\left(H_{C}\right)}>\neq<H_{S W}>$ and $\sigma_{f i t}^{\rho_{F O R C}\left(H_{C}\right)} \neq \sigma_{H_{S w}}$. Similar 
as observed for a mean interaction field, $\left\langle H_{c}^{\rho_{F O R C}\left(H_{c}\right)}\right\rangle$ decreases with $\sigma_{H_{s w}}$ due to the IFD progressive tilting. However, $A, B, C$ points remain reliable indicators for quantitative extraction of the hysteron intrinsic distribution, since $H_{c}^{\text {mean }}$ (taken at point $B$ ) corresponds to $\left\langle H_{S W}\right\rangle$ and $H_{c}^{M H L}$, while $\sigma_{H_{S w}}^{F O R C}=\sigma_{H_{S W}}$. These conclusions are valid for both normal (Table 2) and log-normal SFDs (Table S2).

\begin{tabular}{|c|c|c|c|c|c|}
\hline $\begin{array}{c}<H_{S w}> \\
(\mathrm{mT})\end{array}$ & $\begin{array}{c}\sigma_{H_{S w}} \\
(\mathrm{mT})\end{array}$ & $\begin{array}{c}H_{C}^{\rho_{F O R C}\left(H_{C}\right)} \\
(\mathrm{mT})\end{array}$ & $\begin{array}{c}\sigma_{f i t}^{\rho_{F O R C}\left(H_{C}\right)} \\
(\mathrm{mT})\end{array}$ & $\begin{array}{c}H_{c}^{\text {mean }} \\
(\mathrm{mT})\end{array}$ & $\begin{array}{c}\sigma_{H_{S w}}^{F O R C} \\
(\mathrm{mT})\end{array}$ \\
\hline 300 & 25 & 278 & $39.2(57 \%)$ & $302(0.7 \%)$ & $27.0(8 \%)$ \\
\hline 300 & 40 & 261 & $40.2(0.5 \%)$ & $303(1 \%)$ & $40.4(1 \%)$ \\
\hline 300 & 55 & 249 & $44.9(18 \%)$ & $291(3 \%)$ & $53.4(3 \%)$ \\
\hline 300 & 70 & 238 & $48.6(31 \%)$ & $298(0.7 \%)$ & $70.5(0.7 \%)$ \\
\hline 300 & 85 & 217 & $58.9(31 \%)$ & $309(3 \%)$ & $88.0(3.5 \%)$ \\
\hline
\end{tabular}

Table 2: Main properties (Cf Table 1) extracted from FORC diagrams calculated for hysteron assemblies presenting a normal SFD and interacting in a magnetostatic interaction field (Fig. 7). $\sigma_{H_{S w}}^{F O R C}$ is the SFD deviation deduced from the FORC diagram and Eq. 5.

\section{Interaction field influence}

Previous section reveals that the degree of homogeneity of the interaction field impacts the FORC signature. A mean interaction field leads to constant IFD profiles and wishbone shapes for nonnegligible SFD deviations. Introducing realistic homogeneities, a magnetostatic interaction field yields to peaked IFD profiles and IFD+CFD FORC signatures for dispersed assemblies. In order to link these two interaction regimes, we focus in this part on the influence of the interaction field, particularly in modulating the interaction field deviation $\sigma_{\text {Hint }}$ in the magnetostatic interaction regime.

\section{a. Mean interaction field}

For an assembly of hysterons presenting a large deviation $\left(\sigma_{H_{s w}} / H_{\text {sw }}=30 \%\right)$ of normal (Fig. 8) or log-normal (Fig. S5) SFD, the wishbone shape persists whatever the amplitude of $H_{\text {int }}$. As interaction increases, point $C$ moves toward higher $H_{\mathrm{c}}$ (Fig. 8(f)), Fig. S5(f)). Such a behaviour is coherent with the description given in Sect. $2 \mathrm{a}$ : the wishbone shape, and particularly the point $C$, originate from the enhancement of hysteron widths in the presence of a mean interaction field and SFD. As a result, concerned hysterons move toward higher $H_{\mathrm{c}}$ values in the FORC diagram for increasing $H_{\text {int }}$. The $\rho_{F O R C}$ profiles along the IFD evidence the $H_{\text {int }}$ evolution with an almost constant amplitude (except at point $A$ ), which constitutes the signature of a mean interaction field. For both types of SFD, extraction of quantitative information at points $A$ and $B$ gives the expected $H_{c}^{m e a n}$ and $\sigma_{H_{S w}}^{F O R C}$ values, with errors less than $3 \%$ and $8 \%$, respectively (not shown). 

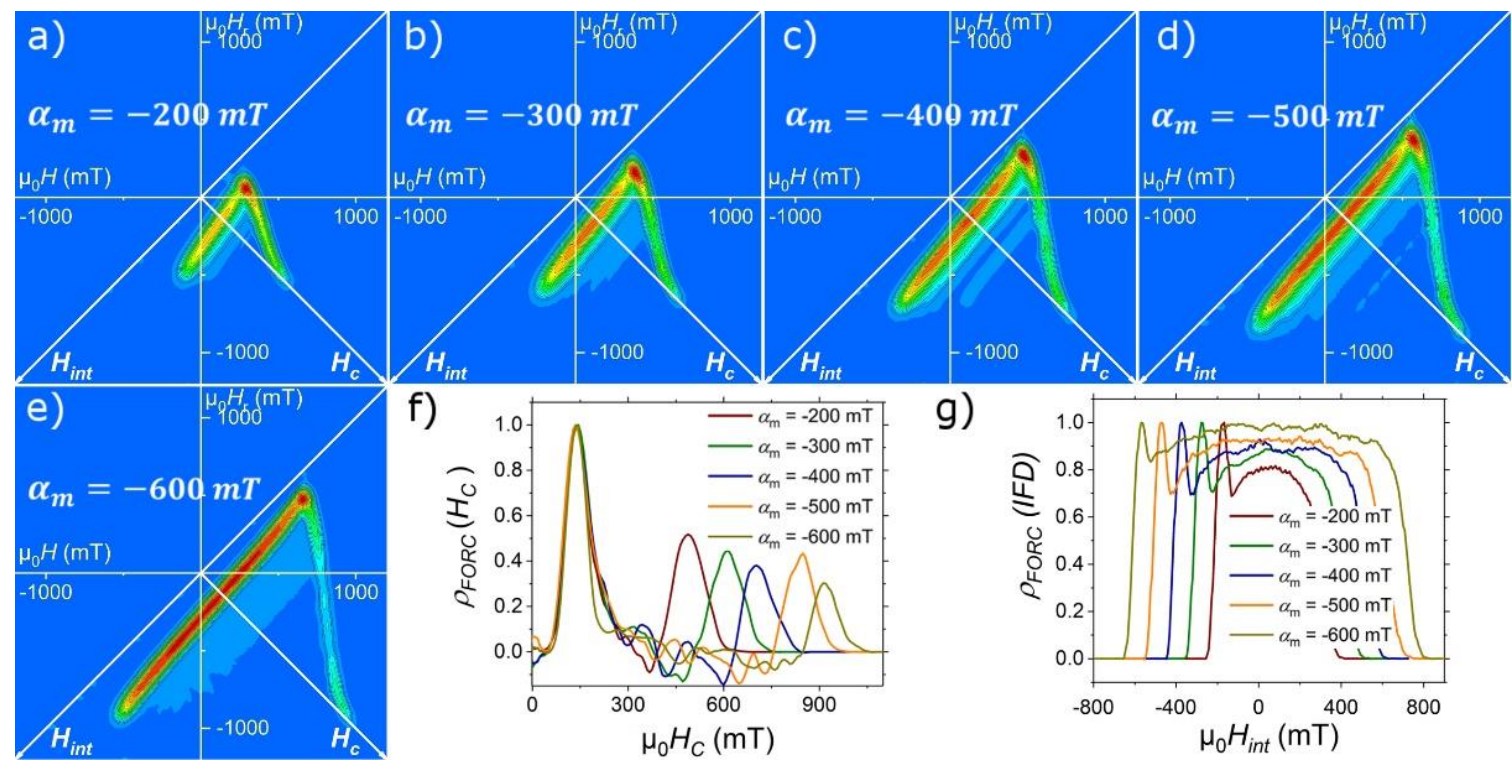

g)

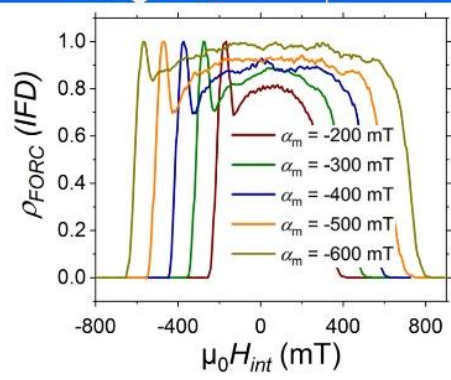

Figure 8: (a)-(e) FORC diagrams calculated for assemblies of hysterons presenting a normal SFD and interacting in a mean interaction field. $\left\langle\mu_{0} H_{s w}\right\rangle=200 \mathrm{mT}, \sigma_{H_{s w}}=60 \mathrm{mT},-600 \leq \alpha_{m} \leq-200 \mathrm{mT}$. (f) $\rho_{F O R C}\left(H_{C}\right)$ and $(\mathrm{g}) \rho_{F O R C}$ profiles along IFD for different $H_{\text {int }}$.

\section{b. Magnetostatic interaction field}

In the present case, the modulation of $H_{\text {int }}$ is realized by varying the distance between hysterons (Eq. 3). For simplicity, we focus on the case of a normal SFD, with $\sigma_{H_{S w}} / H_{S w}=20 \%$. For simulations containing a constant number of hysterons, the assembly size consequently evolves and adds a modulation on $\sigma_{H_{i n t}}$, as small assemblies undergo important edge effects: the hysterons at the periphery present reduced numbers of neighbours and thus experience a smaller interaction field than hysterons located at the assembly centre. Consequently, the increase of $\left\langle H_{\text {int }}\right\rangle$ at low interhysteron distances is concomitant with a $\sigma_{H_{\text {int }}}$ increase.

Figure 9 indicates that FORC diagrams change from a wishbone shape at large inter-hysteron distances to IFD+CFD at low distances. In other words, when $\sigma_{H_{\text {int }}}$ is minimum, at large distances, the interaction regime tends toward a mean field interaction regime for which the FORC signature is a wishbone, whereas at low distances, the interaction regime tends toward an inhomogeneous interaction regime for which the FORC signature is an IFD+CFD. $\rho_{F O R C}$ profiles along IFD (Fig. 9(g)) illustrate this transition in the interaction regime with peaked profiles for low distances and more constant ones for larger distances. Additionally, $\rho_{F O R C}\left(H_{C}\right)$ profiles of Fig. 9(f) show that point $C$ translates to higher $H_{C}$ values at low distances, as a consequence of the $\left\langle H_{\text {int }}\right\rangle$ increase. Quantitative analysis continues to demonstrate that $\rho_{F O R C}\left(H_{C}\right)$ is not directly representative of the SFD, whereas the correct values of $\sigma_{H_{S W}}$ are extracted exploiting $A$ and $B$ points (Table 3 ). 


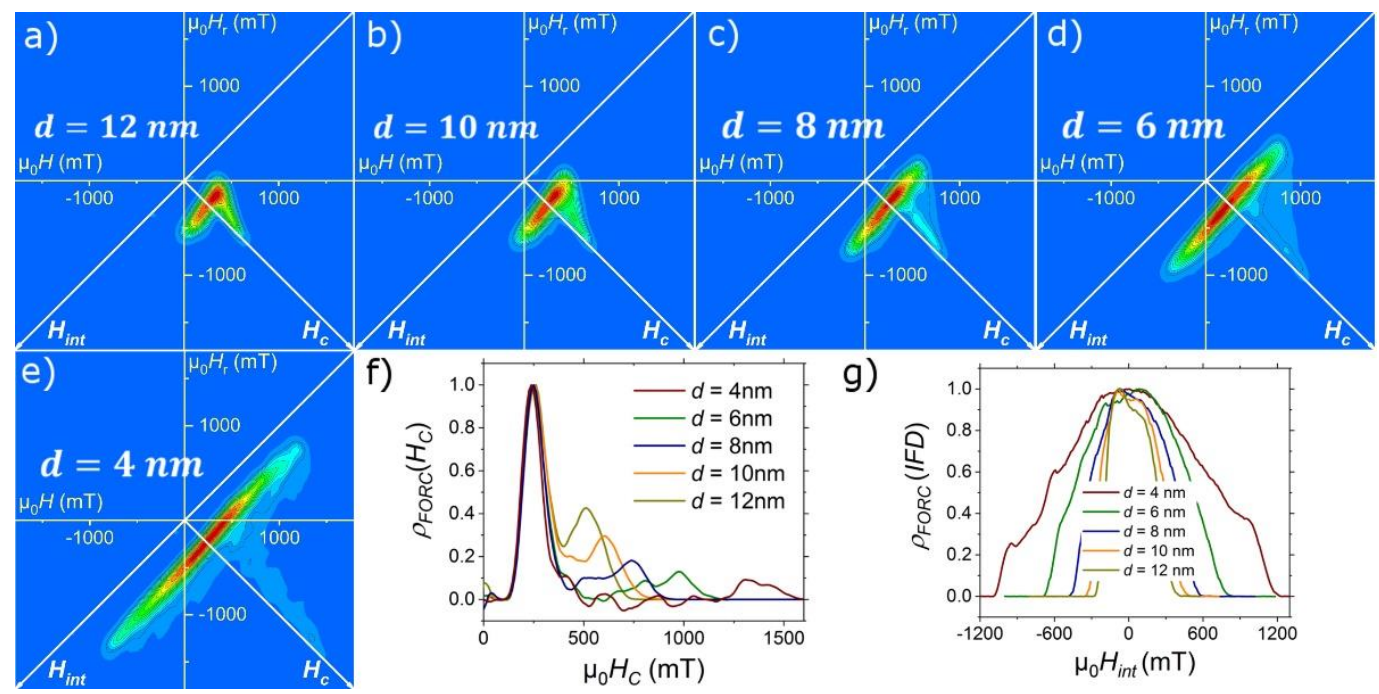

Figure 9: (a)-(e) FORC diagrams calculated for hysteron assemblies presenting a normal SFD with interhysteron distances $4 \leq d \leq 12 \mathrm{~nm}(D=7 \mathrm{~nm})$ and interacting via a magnetostatic interaction field. $N=1681$ hysterons , $<\mu_{0} H_{s w}>=300 \mathrm{mT}, \sigma_{H_{S w}}=60 \mathrm{mT}$. (f) $\rho_{F O R C}\left(H_{c}\right)$ and (g) $\rho_{F O R C}$ profiles along the IFD for the different inter-hysteron distances.

\begin{tabular}{|c|c|c|c|c|c|c|}
\hline $\begin{array}{c}<H_{S w}> \\
(\mathrm{mT})\end{array}$ & $\begin{array}{c}\sigma_{H_{S w}} \\
(\mathrm{mT})\end{array}$ & $\begin{array}{c}d \\
(\mathrm{~nm})\end{array}$ & $\begin{array}{c}H_{C}^{\rho_{F O R C}\left(H_{C}\right)} \\
(\mathrm{mT})\end{array}$ & $\begin{array}{c}\sigma_{f i t}^{\rho_{F O R C}\left(H_{C}\right)} \\
(\mathrm{mT})\end{array}$ & $\begin{array}{c}H_{c}^{\text {mean }} \\
(\mathrm{mT})\end{array}$ & $\begin{array}{c}\sigma_{H_{S w}}^{\text {FORC }} \\
(\mathrm{mT})\end{array}$ \\
\hline 300 & 60 & 12 & 267 & $68.2(13 \%)$ & $302(0.7 \%)$ & $59.6(0.6 \%)$ \\
\hline 300 & 60 & 10 & 263 & $62.4(4 \%)$ & $300(0 \%)$ & $61.6(2.7 \%)$ \\
\hline 300 & 60 & 8 & 253 & $54.1(14 \%)$ & $306(2 \%)$ & $58.6(2.3 \%)$ \\
\hline 300 & 60 & 6 & 253 & $54.4(9 \%)$ & $296(1.3 \%)$ & $58.7(2.2 \%)$ \\
\hline 300 & 60 & 4 & 242 & $48.4(19 \%)$ & $302(0.7 \%)$ & $58.3(2.8 \%)$ \\
\hline
\end{tabular}

Table 3: Main properties (Cf Table 1) extracted from FORC diagrams of Fig. 9, calculated for assemblies of hysterons with edge-to-edge inter-hysteron distances $4 \leq d \leq 12 \mathrm{~nm}(D=7 \mathrm{~nm})$ in a magnetostatic interaction field.

Data of Fig. 9 indicate that a transition between wishbone and IFD+CFD shapes is obtained when both $\left\langle H_{\text {int }}>\right.$ and $\sigma_{H_{\text {int }}}$ increase. In order to modulate $\sigma_{H_{\text {int }}}$ independently, the size of the hysteron assembly can be modified by varying the inter-hysteron distance $d$, but keeping $\left\langle H_{\text {int }}\right\rangle$ constant by adjusting accordingly the diameter $D$ of the nanowire-based hysteron (Eq. 3). Therefore, $\sigma_{H_{\text {int }}}$ is calculated as the mean deviation of the averaged sum of interaction field to the one of each hysteron: $\sigma_{H_{i n t}}=\sum_{i=1}^{N} \frac{\sqrt{\left(<H_{i n t}>-H_{i n t}^{i}\right)^{2}}}{N}$, with $<H_{\text {int }}>=\frac{1}{N} \sum_{i=1}^{N} H_{i n t}^{i}$ and $H_{i n t}^{i}$ the interaction field of the $i^{\text {th }}$ hysteron calculated using Eq. 3 . Note that $\sigma_{H_{\text {int }}}$ is here tuned by varying the assembly size, but it can also be done alternatively introducing dispersions in $D, L$ or $d$ in Eq. 3, which are the real origins of $\sigma_{H_{\text {int }}}$ in practice.

In order to compare small vs large inter-hysteron distances $d$, the diameter $D$ is adjusted to yield the same calculated $\left.<H_{\text {int }}\right\rangle$ value and this whatever the magnetization state (Fig. 10(a) for three $<$ $H_{\text {int }}>$ values). Corresponding $\sigma_{H_{\text {int }}}$ is plotted as a function of applied field in Fig. 10(b). Figure 11 plots the corresponding FORC diagrams to compare small vs large inter-hysteron distances which give the same $\left\langle H_{\text {int }}\right\rangle$. At low inter-hysteron distances, i.e. for small assemblies, the saturation value of $\sigma_{H_{i n t}}$ in Fig. 10 (b) reaches highest values as a result of edge effects. Indeed, for large inter-hysteron 
distances, saturation values of $\sigma_{H_{\text {int }}}$ are comparatively lower. An important contrast is that $\sigma_{H_{\text {int }}}$ is constant during the magnetization reversal for large inter-hysteron distances $(d=50 \mathrm{~nm})$, which lead to wishbone FORC diagrams (Figs. 11 (d)-(e)-(f)). It illustrates the fact that in this case, the switching of hysterons is not guided by the interaction field, but solely by the SFD. Indeed, if the demagnetizing interaction field would drive the magnetization reversal, areas with large local interaction fields would be favoured and consecutive switchings would lead to a local decrease of $H_{\text {int }}: \sigma_{H_{\text {int }}}$ should strongly vary to reach a minimum at $H_{c}$. This behaviour is observed in Fig. 10(b) for small inter-hysteron distances, which present IFD+CFD FORC diagrams (Figs. 11 (a)-(b)-(c)). In that case, $\sigma_{H_{\text {int }}}$ is minimum in the demagnetized state, at $H_{c}$, and maximum at saturation, in agreement with previous results [5]. Therefore, in the case of a highly dispersed interaction field, the reversal is guided by the nonuniformity of the interaction field, whereas for less dispersed interactions, the reversal tends to be guided by the SFD.
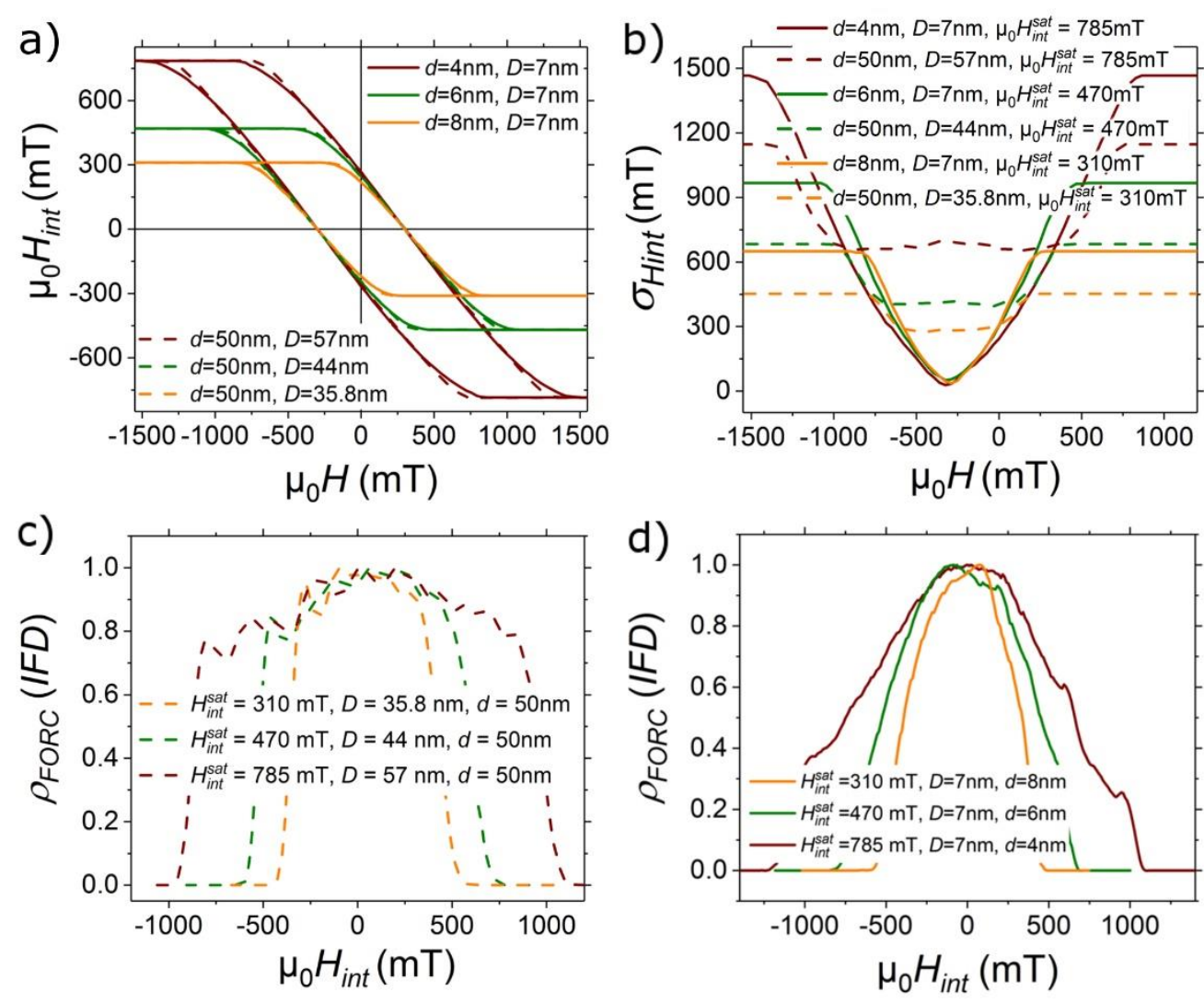

Figure $10:$ a) Interaction field averaged on the whole hysterons and b) standard deviation of the interaction field $\sigma_{H_{i n t}}$, plotted as a function of applied field. c) $\rho_{F O R C}$ profiles along the IFD for inter-hysteron distance $d=50 \mathrm{~nm}$ (FORC diagrams of Fig. 11(d)-(e)-(f)). d) $\rho_{F O R C}$ profiles along the IFD for diameter of nanowirehysteron $D=7 \mathrm{~nm}$ (FORC diagrams of Fig. 11(a)-(b)-(c)). $H_{\text {int }}^{\text {sat }}$ corresponds to the averaged saturation value of $H_{\text {int }}$ over the assembly and is obtained from Fig. 10(a). 

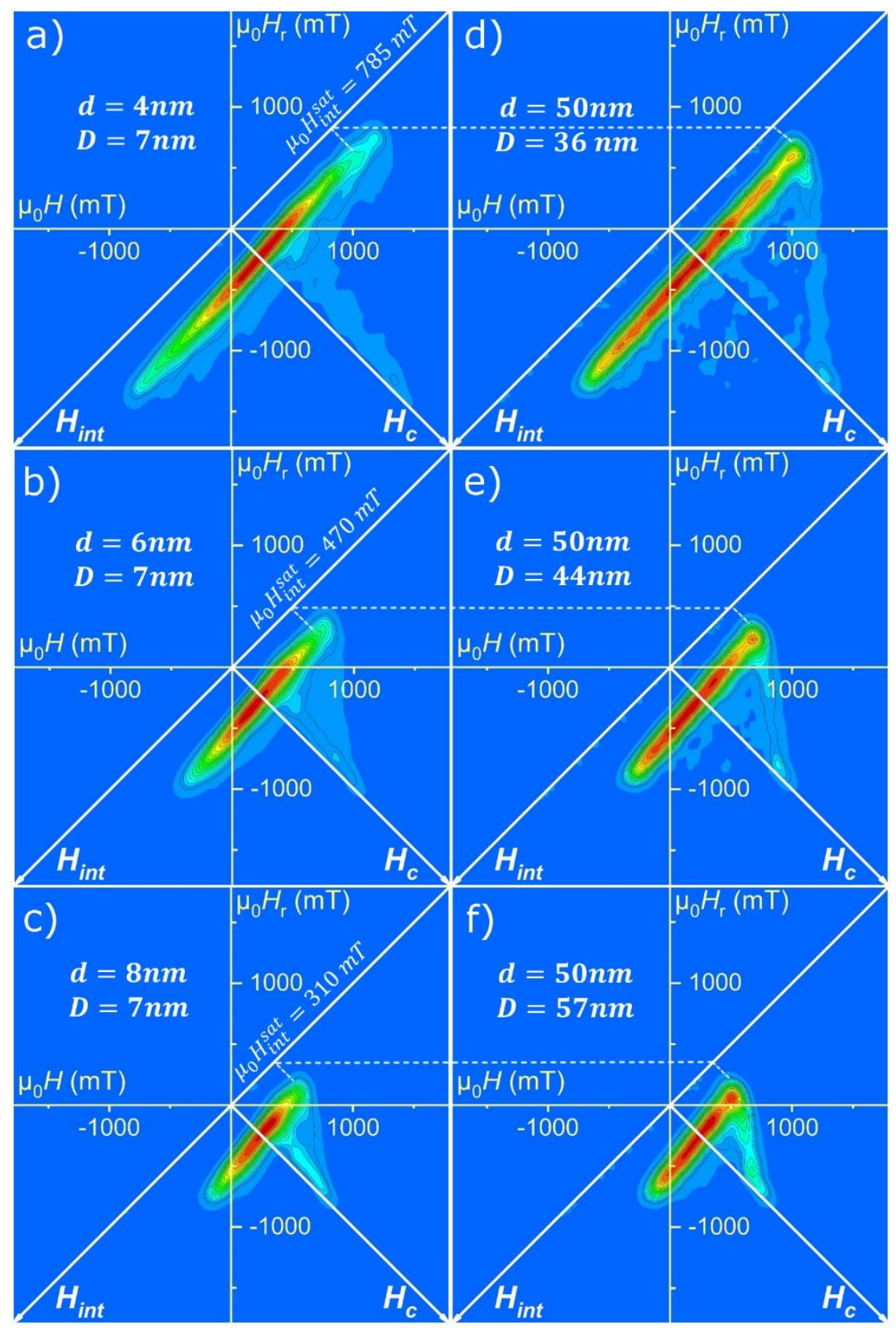

Figure 11: (a)-(c) FORC diagrams calculated for hysteron assemblies with edge-to-edge inter-hysteron distances $4 \leq d \leq 6 \mathrm{~nm}$ and diameter $D=7 \mathrm{~nm}$. (d)-(f) FORC diagrams calculated for hysteron assemblies with inter-hysteron distances $d=50 \mathrm{~nm}$. The diameter $D$ of the nanowires whose hysterons referred to has been modulated within $35.8 \leq D \leq 57 \mathrm{~nm}$ to obtain $\left\langle H_{\text {int }}>\right.$ identical to the FORC diagrams (a)-(c). $N=1681$ hysterons, $<\mu_{0} H_{s w}>=300 \mathrm{mT}, \sigma_{H_{S w}}=60 \mathrm{mT}$. $H_{\text {int }}^{\text {sat }}$ values are obtained from Fig. 10(a) and are here located on FORC diagrams for illustration.

The IFD profiles are almost constant for large inter-hysteron distances (Fig. 10(c)) whereas they are sharped for small ones (Fig. 10(d)). As seen in previous parts, this difference has been ascribed to mean-field and local interaction field, respectively. Assemblies with large inter-hysteron distances, exhibiting low $\sigma_{H_{\text {int }}}$ at saturation, behave as in a mean-field regime and a wishbone type FORC diagram is obtained, provided that a SFD is present. On the other side, low distance assemblies, exhibiting high $\sigma_{H_{i n t}}$ at saturation, behave as in a local interaction field regime and the FORC signature is IFD+CFD. Here we can conclude that, among constant parameters $\left(H_{\text {int }}, H_{S W}, \sigma_{H_{S w}}\right), \sigma_{H_{\text {int }}}$ is an important 
parameter that drives the FORC signature. Nevertheless, the quantitative determination of $\sigma_{H_{\text {int }}}$ from the FORC diagram is not accessible.

\section{Discussion}

The simulations presented here allow to distinguish three major FORC signatures that can be encountered in assemblies of perpendicularly magnetized nanomagnets described by rectangular hysterons in the presence of demagnetizing dipolar interactions: linear (or IFD), wishbone, and IFD+CFD.

First, the linear FORC is obtained for hysteron assemblies presenting no or extremely small switching-field distributions. This signature consists in the interaction field distribution (IFD) entirely due to the sheared hysteresis loop in the presence of demagnetizing dipolar interactions. IFD is parallel to the $H_{\text {int }}$ axis and the profile is constant for a mean interaction field, whereas it is peaked for a magnetostatic interaction field with a maximum at $H_{\text {int }}=0$. This feature can constitute a criterion to distinguish the interaction regime. For both regime of interactions, the IFD width is $2 H_{\text {int }}^{\text {sat }}$, thus $H_{\text {int }}^{\text {sat }}$ is easily accessible typically at point $A$. The coercive field, i.e. the median switching field, can be extracted at point $B$ because its abscissa is always $H_{c}^{\text {median }}=\left\langle H_{S W}\right\rangle$. Modulations of $\left\langle H_{s w}\right\rangle$ or amplitude of $H_{\text {int }}$ do not affect the FORC signature and only shift the $\rho_{F O R C}$ distributions. If a (low) SFD is present, the $\rho_{F O R C}\left(H_{C}\right)$ profile does not quantitatively represent it. Nevertheless, it can be successfully addressed by $\sigma_{H_{s w}}^{F O R C}$ determined from $H_{c}^{\text {min }}$ and $H_{c}^{\text {median }}$ extracted at points $A$ and $B$, respectively, and this whatever the SFD shape.

Secondly, the wishbone FORC is clearly obtained for hysteron assemblies presenting a switching-field distribution and where a uniform interaction field, i.e. mean-field, dominates. The $A C$ branch of the wishbone shape originates from the most coercive hysterons of a given FORC : they undergo a bias $H_{u}$ proportional to the $f_{1}$ proportion of up hysterons, together with an artificial increase of their width $2 H_{c}$ proportionally to the $f_{2}$ proportion of down hysterons. IFD occurs due to the sheared hysteresis loop and accounts for the bias field underwent by the less coercitive hysterons. As a consequence, the tilt between IFD and $H_{\text {int }}$ axis increases with the SFD deviation $\sigma_{H_{s w}}$. $H_{c}^{\text {median }}=$ $<H_{S W}>$ and $H_{\text {int }}^{\text {sat }}$ can be read at point $B$ and point $A$ respectively. Whereas $\rho_{F O R C}\left(H_{C}\right)$ profile does not quantitatively account for SFD, $\sigma_{H_{s w}}$ can be also obtained using abscissa of points $A$ and $B$.

Finally, the IFD+CFD FORC signature is mainly composed of the IFD, which is still due to the $\mathrm{MHL}$ shearing. Similarly to the precedent cases, the interaction field value at saturation $H_{\text {int }}^{\text {sat }}$ can also be obtained at point $A$, while point $B$ indicates $H_{c}^{\text {median }}=\left\langle H_{S w}\right\rangle$. Since CFD is being mostly aligned with $H_{c}$ axis (i.e. $H_{\text {int }}=0$ ), it concerns symmetrical or quasi-symmetrical hysterons: it occurs for assemblies of hysterons interacting via a local interaction field with a significant deviation $\sigma_{H_{i n t}}$. For large deviations, $\sigma_{H_{\text {int }}}$ evolves strongly during the magnetization reversal, the non-uniform interaction regime gives peaked $\rho_{F O R C}(I F D)$ profiles with maximum at $H_{\text {int }}=0$ and the FORC diagram is IFD+CFD. Conversely, for small deviations, $\sigma_{H_{\text {int }}}$ does not strongly change with $M$, the interaction field tends to be homogenous with constant $\rho_{F O R C}$ profiles along IFD and the FORC diagram is a wishbone. Whatever the IFD+CFD or wishbone shape, deviation of the switching field distribution can be quantified with error less than $10 \%$ using abscissa of points $A$ and $B$, provided that the studied system corresponds to isolated coupled elements with only demagnetizing dipolar interactions.

From the experimental point of view, as quantitative data are contained in points $A, B$ and $C$, it is crucial to accurately locate these points on the FORC diagrams. Point $A$ and $B$ can be determined 
moving horizontal lines parallel to the $H$ axis and intercepting extremal values of the IFD where $\rho_{\text {FORC }} \neq 0$ (Fig. 6). Point $C$, in principle horizontally aligned with point $B$, is located on the $H_{C}$ axis where $\rho_{F O R C}$ reaches the highest $H_{C}$ value. In order to try to access the SFD shape, a deshearing process [9] can be done if the FORC diagram reveals that the interaction regime is uniform, as attested by the IFD profile and/or the presence of a wishbone shape. In that case, $H_{\text {int }}^{\text {sat }}$ determined at point $A$ can be used to evaluate $\alpha_{m}$ and plotting the FORCs as a function of $H_{\text {tot }}$ instead of $H_{\text {ext }}$ using Eq. 4. SFD can then be obtained differentiating the MHL or using $\rho_{F O R C}\left(H_{c}\right)$ profile. Nevertheless, deshearing is non trivial in practice, as it can often lead to overskewing due to the inherent assumptions associated with such an approach [69].

\section{Conclusion}

Main signatures of FORC diagrams encountered in 2D arrays of perpendicularly magnetized nanomagnets in the presence of demagnetizing dipolar interactions are reproduced and analysed with the sake to propose their quantified reading. For systems satisfying the present model, interaction regime together with its amplitude, as well as mean coercivity and SFD deviation, can be obtained, even if the SFD shape in itself cannot be determined. Next step is to overcome some of the assumptions, for example in introducing a magnetizing coupling between magnets, such as exchange interaction or magnetizing dipolar interactions in assemblies where aligned easy axes lie in the assembly plane. In addition to the extraction of extrinsic and intrinsic parameters, FORC is also an exciting tool to probe fingerprints related to the reversal processes such as distributions related to nucleation/annihilation of magnetic domain in thin films [18][19] or vortex in dots [25]. Such behaviours cannot be addressed using the hysteron-based approach and thus require FORC modelling with the use of micromagnetic simulations, which are up to now time-consuming and limits a broad scanning of parameters.

\section{Supplementary Material}

See supplementary material for supplementary figures and tables, description of the origin of the FORC diagram wishbone shape, and extraction of the standard deviation of a log-normal distribution from the FORC diagram.

\section{Acknowledgements}

This work was supported by the Agence Nationale de la Recherche (France) under contract No. ANR14-CE07-0025-01 (DENSAR) and through the EUR grant NanoX n ${ }^{\circ}$ ANR-17-EURE-0009 in the framework of the "Programme des Investissements d'Avenir".

\section{Data availability}

The data that support the findings of this study are available from the corresponding author upon reasonable request.

\section{References}

[1] I. D. Mayergoyz, Phys. Rev. Lett. 56, 1518 (1986). 
[2] F. Preisach, Z. Phys. 94, 277 (1935).

[3] A.P. Roberts, D. Heslop, X. Zhao, C.R. Pike, Rev. Geophys. 52, 557-602 (2014).

[4] C.R. Pike, A.P. Roberts, K.L. Verosub, J. Appl. Phys. 85, 6660 (1999).

[5] C.-I. Dobrotă, A. Stancu, J. Appl. Phys. 113, 043928 (2013).

[6] C. Carvallo, A. Muxworthy, Geochem. Geophys. Geosyst. 7, Q09003 (2006).

[7] S.-C. L.L. Lappe, J.M. Feinberg, A. Muxworthy, R.J. Harrison, Geochem. Geophys. Geosyst. 14, 2143 (2013).

[8] X. Zhao, A. P. Roberts, D. Heslop, G.A. Paterson, Y. Li, J. Li, J. Geophys. Res. Solid Earth 122, 4767 (2017).

[9] C. Papusoi, K. Srinivasan, R. Acharya, J. Appl. Phys 110, 083908 (2011).

[10] C. Papusoi, M. Desai, R. Acharya, J. Phys. D: Appl. Phys. 48, 215005 (2015).

[11] S. N. Piramanayagam, M. Ranjbar, J. Appl. Phys 111, $07 B 728$ (2012).

[12] H. K. Tan, B. Varghese, S.N. Piramanayagam, J. Appl. Phys 116, 163909 (2014).

[13] R. K. Dumas,P.K. Greene,D.A. Gilbert,L. Ye,C. Zha, J. Åkerman, K. Liu, Phys. Rev. B 90, 104410 (2014).

[14] J.B. Abugri, P.B. Visscher, S. Gupta, P. J. Chen, R. D. Shull, J. Appl. Phys. 124, 043901 (2018).

[15] D.R. Cornejoa, R.D. Noceb, T.R.F. Peixotoa, N. Barelli, P.T.A. Sumodjoc, A.V. Benedetti, J. Alloys Compd. 479, 43 (2009).

[16] Y. Cao, K. Xua, W. Jiang, T. Droubay, P. Ramuhalli, D. Edwards, B.R. Johnson, J. McCloy, J. Magn. Magn. Mater. 395, 361 (2015).

[17] C. Papusoi, S. Jain, R. Admana, B. Ozdol, C. Ophus, M. Desai, R. Acharya, J. Phys. D: Appl. Phys. 50,285003 (2017).

[18] D. Navas, N. Soriano, F. Béron, C.T. Sousa, K.R. Pirota, J. Torrejon, C. Redondo, R. Morales, C.A. Ross, Phys. Rev. B 96, 180403(R) (2017).

[19] J. E. Davies, O. Hellwig, E.E. Fullerton, G. Denbeaux, J. B. Kortright, K. Liu, Phys. Rev. B 70, 224434 (2004). 
[20] J. E. Davies, O. Hellwig, E.E. Fullerton, K. Liu, Phys. Rev. B 77, 014421 (2008).

[21] J. Yin, H. Zhang, F. Hu, B. Shen, L.Q. Pan, J. Appl. Phys. 106, 103901 (2009).

[22] J. E. Davies, O. Hellwig, E. E. Fullerton, M. Winklhofer, R. D. Shull, Kai Liu, Appl. Phys. Lett. 95, 022505 (2009).

[23] A. Markou, I. Panagiotopoulos, T. Bakas, P. Postolache, L. Stoleriu, and A. Stancu, J. Appl. Phys. 112, 123914 (2012).

[24] Z. Diao, N. Decorde, P. Stamenov, K. Rode, G. Feng, J. M. D. Coey, J. Appl. Phys. 111, 07B538 (2012).

[25] R.K. Dumas, C.-P. Li, I.V. Roshchin, I.K. Schuller, K. Liu, Phys. Rev. B 75, 134405 (2007).

[26] C.R. Pike, C.A. Ross, R.T. Scalettar, G. Zimanyi, Phys. Rev. B 71134407 (2005).

[27] D.A. Gilbert, G.T. Zimanyi, R.K. Dumas, M. Winklhofer, A. Gomez, N. Eibagi, J. L. Vicent, K. Liu, Sci. Rep. 4, 4204 (2014).

[28] M. Ito, M. Yano, N. Sakuma, H. Kishimoto, A. Manabe, T. Shoji, A. Kato, N. M. Dempsey, D. Givord, G.T. Zimanyi, AIP Adv. 6, 056029 (2016).

[29] S. Muralidhar, J. Gräfe, Y.-C. Chen, M. Etter, G. Gregori, S. Ener, S. Sawatzki, K. Hono, O. Gutfleisch, H. Kronmüller, G. Schütz, E.J. Goering, Phys. Rev. B 95, 024413 (2017).

[30] J.E. Davies, O. Hellwig, E.E. Fullerton, J.S. Jiang, S.D. Bader, G.T. Zimányi, K.Liu, Appl. Phys. Lett. 86, 262503 (2005).

[31] L. Spinu, A. Stancu, C. Radu, F. Li, J.B. Wiley, IEEE Trans. Magn. 40, 2116 (2004).

[32] M. Ciureanu, F. Béron, P. Ciureanu, R. W. Cochrane, D. Ménard, A. Sklyuyev, A. Yelon, J. Nanosci. Nanotechnol. 8, 5725 (2008).

[33] A. Rotaru, J.-H. Lim, D. Lenormand, A. Diaconu, J.B. Wiley, P. Postolache, A. Stancu, L. Spinu, Phys. Rev. B 84 , 134431 (2011).

[34] M.P. Proenca, C. T. Sousa, J. Ventura, J. Garcia, M. Vazquez, J.P. Araujo, J. Alloys Compd. 699, 421e429(2017).

[35] M. Nica, A. Stancu, Physica B 475, 73 (2015). 
[36] M.P. Proenca, J. Ventura, C.T. Sousa, M. Vazquez, J.P. Araujo, J. Phys.: Condens. Matter 26 116004 (2014).

[37] A.H. Montazer, A. Ramazani, M. Almasi Kashi, J. Zavašnik, J. Phys. D: Appl. Phys. 49445001 (2016).

[38] D. Cimpoesu, I. Dumitru, A. Stancu, J. App. Phys. 120, 173902 (2016).

[39] R. Lavin, J.C. Denardin, J. Escrig, D. Altbir, A. Cortes, H. Gomez, IEEE Trans. Magn. 44(11) 2808 (2008).

[40] M. P. Proenca, C.T. Sousa, J. Escrig, J. Ventura, M. Vazquez, and J. P. Araujo, J. App. Phys. 113, 093907 (2013).

[41] E.M. Palmero, F. Béron, C. Bran, R.P del Real, M. Vázquez, Nanotechnology 27, 435705 (2016).

[42] P. Sergelius, J. G. Fernandez, S. Martens, M. Zocher, T. Böhnert, V. V. Martinez, V. Manuel de la Prida, D. Görlitz, K. Nielsch, J. Phys. D: Appl. Phys. 49(14), 145005 (2016).

[43] F. Béron, D. Ménard, A. Yelon, J. App. Phys. 103, 07D908 (2008).

[44] A. Stancu, C. Pike, L. Stoleriu, P. Postolache, D. Cimpoesu, J. App. Phys. 93, 6620 (2003).

[45] A. Muxworthy, W. Williams, J. App. Phys. 97, 063905 (2005).

[46] A. Muxworthy, D. Heslop, W. Williams, Geophys. J. Int. 158, 888 (2004).

[47] C.-I. Dobrotă, A. Stancu, Physica B 4074676 (2012).

[48] C.-I. Dobrotă, A. Stancu, J. Phys. Cond. Mat. 25, 035302(2013).

[49] C.-I. Dobrotă, A. Stancu, Physica B 457, 280 (2015).

[50] A. Berger, B. Lengsfield, Y. Ikeda, J. Appl. Phys. 99, 08 E705 (2006)

[51] J. M. Martínez Huerta, J. De La Torre Medina, L. Piraux, A. Encinas, J. Appl. Phys. 111, 083914 (2012).

[52] M. Winklhofer, G.T. Zimanyi, J. App. Phys. 99, 08 E710 (2006).

[53] S. Ruta, O. Hovorka, P.-W. Huang, K. Wang, G. Ju, R. Chantrell, Sci. Rep. 7, 45218 (2017).

[54] E. Della Torre, IEEE Trans. Audio Electroacoust. 14, 86 (1966). 
[55] L.-P. Carignan, C. Lacroix, A. Ouimet, M. Ciureanu, A. Yelon, D. Ménard, J. Appl. Phys. 102023905 (2007).

[56] F. Béron, D. Ménard, A. Yelon, J. Appl. Phys. 103(7), 07D908 (2008).

[57] R. Lavin, J.C. Denardin, J. Escrig, D. Altbir, A. Cortes, H. Gomez, IEEE Trans. Magn. 44(11), 2808 (2008).

[58] S. Samanifar, M. A. Kashi, A. Ramazani, Phys. C Supercond. Its Appl. 548, 72 (2018).

[59] X. Kou, X. Fan, R.K. Dumas, Q. Lu , Y. Zhang, H. Zhu, X. Zhang, K. Liu, J.Q. Xiao, Adv. Mater. 23(11), 1393 (2011).

[60] M.P. Proenca, C.T. Sousa, J. Ventura, J. Garcia, M. Vazquez, J.P. Araujo, J. Alloys Compd., 699, 421 (2017).

[61] L.C.C. Arzuza, R. López-Ruiz, D. Salazar-Aravena, M. Knobel, F. Béron, K.R. Pirota, J. Magn. Magn. Mater. 432, 309 (2017).

[62] A. Ramazani, V. Asgari, A. H. Montazer, M. Almasi Kashi, Curr. Appl. Phys., 15(7), 819 (2015).

[63] V. Vega et al., J. Nanosci. Nanotechnol. 12(6), 4736 (2012).

[64] T.R.F. Peixoto, D.R. Cornejo, J. Magn. Magn. Mater. 320(14), e279 (2008).

[65] M.P. Proenca, C.T. Sousa, J. Escrig, J. Ventura, M. Vazquez, J.P. Araujo, J. Appl. Phys. 113(9), 093907 (2013).

[66] F. Béron, L. Clime, M. Ciureanu, D. Ménard, R.W. Cochrane, A. Yelon, J. Nanosci. Nanotechnol. 8(6), 2944 (2008).

[67] M.R.Z. Kouhpanji, B.J.H. Stadler, Nano Express 1, 010017 (2020)

[68] D.A. Gilbert, G.T. Zimanyi, R.K Dumas, M. Winklhofer, A. Gomez, N. Eibagi, J.L. Vicent, K. Liu, Sci. Rep .4, 4204 (2014)

[69] R. Skomski, J.P. Liu, D.J. Sellmyer, Phys. Rev. B 60(10), 7359 (1999). 